\title{
Aging Wireless Bandits: Regret Analysis and Order-Optimal Learning Algorithm
}

\author{
Eray Unsal Atay \\ Bilkent University, Ankara, Turkey \\ eray.atay@ug.bilkent.edu.tr
}

\author{
Igor Kadota \\ Columbia University, New York, USA \\ igor.kadota@columbia.edu
}

\author{
Eytan Modiano \\ MIT LIDS, Cambridge, USA \\ modiano@mit.edu
}

\begin{abstract}
We consider a single-hop wireless network with sources transmitting time-sensitive information to the destination over multiple unreliable channels. Packets from each source are generated according to a stochastic process with known statistics and the state of each wireless channel (ON/OFF) varies according to a stochastic process with unknown statistics. The reliability of the wireless channels is to be learned through observation. At every time-slot, the learning algorithm selects a single pair (source, channel) and the selected source attempts to transmit its packet via the selected channel. The probability of a successful transmission to the destination depends on the reliability of the selected channel. The goal of the learning algorithm is to minimize the Age-of-Information (AoI) in the network over $T$ time-slots. To analyze its performance, we introduce the notion of AoI-regret, which is the difference between the expected cumulative AoI of the learning algorithm under consideration and the expected cumulative AoI of a genie algorithm that knows the reliability of the channels a priori. The AoI-regret captures the penalty incurred by having to learn the statistics of the channels over the $T$ time-slots. The results are two-fold: first, we consider learning algorithms that employ well-known solutions to the stochastic multi-armed bandit problem (such as $\epsilon$-Greedy, Upper Confidence Bound, and Thompson Sampling) and show that their AoI-regret scales as $\Theta(\log T)$; second, we develop a novel learning algorithm and show that it has $O(1)$ regret. To the best of our knowledge, this is the first learning algorithm with bounded AoI-regret.
\end{abstract}

Index Terms-Age of Information, Wireless Networks, Regret, Multi-Armed Bandits, Learning

\section{INTRODUCTION}

Age-of-Information (AoI) is a performance metric that captures the freshness of the information from the perspective of the destination. AoI measures the time that elapsed since the generation of the packet that was most recently delivered to the destination. This performance metric has been receiving attention in the literature [1], [2] for its application in communication systems that carry time-sensitive data. In this paper, we consider a network with $M$ sources transmitting time-sensitive information to the destination over $N$ unreliable wireless channels, as illustrated in Fig. 1. Packets from each source are generated according to an i.i.d. stochastic process with known statistics and the state of each wireless channel (ON/OFF) varies according to an i.i.d. stochastic process with unknown statistics. At every time-slot, the learning algorithm schedules a single pair (source, channel) and the selected source attempts to transmit its packet via the selected wireless channel. When a packet with fresh information is successfully transmitted to the destination, the AoI associated with the selected source is reduced. The goal of the learning algorithm is to keep the information associated with every source in the network as fresh as possible, i.e. to minimize the AoI in the network. To decide which pair (source, channel) to select in a time-slot, the learning algorithm takes into account: i) the packet generation processes at the $M$ sources; ii) the current value of AoI associated with each of the $M$ sources; and iii) the estimated reliability of the $N$ wireless channels.

In this sequential decision problem, the outcomes of previous transmission attempts are used to estimate the reliability of the wireless channels. This statistical learning problem is closely related to the stochastic multi-armed bandit (MAB) problem in which the wireless channels are the bandits that give i.i.d. rewards and the learning algorithm is the player that attempts to learn the statistics of the bandits in order to maximize the reward accumulated over time. The main challenge in the stochastic MAB problem is to strike a balance between exploiting the bandit that gave the highest rewards in the past and exploring other bandits that may give high rewards in the future. To evaluate the performance of different learning algorithms, we define regret. Regret is the difference between the expected cumulative reward of a genie algorithm (that knows the statistics of the bandits a priori) and the expected cumulative reward of the learning algorithm under consideration. The regret captures the penalty incurred by having to learn the statistics of the bandits over time. Some well-known order-optimal learning algorithms in terms of regret are: $\epsilon$-Greedy, Upper Confidence Bound (UCB), and Thompson Sampling (TS). The regret of these policies was shown to increase no more than logarithmically in time [3][5], $O(\log T)$, and this bound was shown to be tight [6].

We refer to our problem as the Aging Bandit problem. An important distinction between the stochastic MAB problem and the Aging Bandit problem is the reward structure. In the stochastic MAB problem, the player selects a bandit in each time-slot and receives a reward that is i.i.d. over time and depends only on the probability distribution associated with the selected bandit. In the Aging Bandit problem, the learning algorithm selects a pair (source, channel) and the reward is the AoI reduction that results from a packet transmission to the destination. This reward depends on the state of the selected channel (which is i.i.d. over time), since a failed transmission gives zero reward, and it also depends on the 
history of previous packet deliveries and packet generations. In particular, if the selected source has recently delivered a fresh information update to the destination, then the reduction in AoI may be small. In contrast, if the selected source has not updated the destination for a long period, then the AoI reduction may be large. The reward structure of Aging Bandits is closely related to the AoI evolution (formally defined in Sec. II) which is history-dependent. This intricate reward structure has significant impact on the analysis of regret and on the development of learning algorithms when compared to the analysis of the traditional stochastic MAB.

The literature on MAB problems is vast, dating more than eight decades [7]. For surveys on different types of $\mathrm{MAB}$ problems, we refer the readers to [8], [9]. Most relevant to this work are [10]-[20]. The authors in [10]-[12] considered the problem of minimizing the expected queue-length in a system with a single queue and multiple servers with unknown service rates. In [10], the authors introduced the concept of queue-length regret, developed a learning algorithm inspired by Thompson Sampling, and analyzed its regret. In [11], [12], the authors used information particular to the queue evolution to develop a learning algorithm with $O(1)$ queue-length regret.

The authors in [13]-[20] considered the problem of minimizing the average AoI in a single-hop wireless network with unreliable channels. In [13]-[17], the authors posed the AoI minimization problem in a network with multiple sources and known channel statistics as a restless MAB problem, developed the associated Whittle's Index scheduling policy, and evaluated its performance in terms of the average AoI. In [18], the authors considered the AoI minimization problem in a network with a single source-destination pair and unknown channel statistics, introduced the concept of AoI-regret, and showed that the regret of UCB and TS scale as $O(\log T)$. In [19], the authors obtained similar results as in [18] for the more challenging case of correlated wireless channels. In [20], the authors considered the AoI minimization problem in a network with multiple sources that generate and transmit fresh packets at every time-slot through (possibly) different channels with unknown statistics. The authors in [20] show that the regret of a UCB-based distributed learning algorithm scales as $O\left(\log ^{2} T\right)$. An important modelling assumption common to [18]-[20] is that sources generate and transmit fresh packets in every time-slot. The more realistic assumptions of stochastic packet generation and scheduled transmissions have significant impact on the AoI evolution, on the analysis of AoI-regret, and on the development of learning algorithms. For example, in Sec. IV, we leverage the random packet generation to develop (for the first time) a learning algorithm with $O(1)$ AoI-regret.

In this paper, we study learning algorithms that attempt to minimize AoI in a network with multiple sources generating packets according to stochastic processes and transmitting these packets to the destination over wireless channels with initially unknown statistics. At every time-slot, the learning algorithm schedules a single pair (source, channel) and the selected source attempts to transmit a packet in the selected channel. Note that the source policy, which selects the source

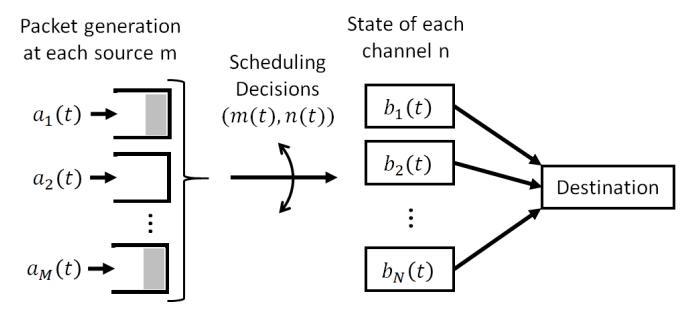

Fig. 1. Wireless network with $M$ sources, $N$ channels, and destination.

at each time-slot, and the channel policy, which selects the channel to be used in each time-slot, can be naturally separated. In this paper, we focus on the exploration-exploitation dilemma faced by the channel policy. In particular, we consider learning algorithms employing the optimal source policy and different channel policies. Our main contributions include:

- we analyze the performance of channel policies based on traditional MAB algorithms including $\epsilon$-Greedy, UCB, and TS, and show that their AoI-regret scales as $\Theta(\log T)$. These results generalize the analysis in [18] to networks with multiple sources generating packets randomly. The analysis of the AoI-regret is more challenging in this network setting since the AoI evolution depends on both the source policy and the stochastic packet generation process. These challenges are discussed in Sec. III;

- we develop a novel learning algorithm and establish that it has $O(1)$ AoI-regret. The key insight is that when packets are generated randomly, the learning algorithm can utilize times when all $M$ transmission queues are empty to transmit dummy packets and learn the statistics of the channels without incurring a cost in terms of AoIregret. To the best of our knowledge, this is the first learning algorithm with bounded AoI-regret.

The remainder of this paper is outlined as follows. In Sec. II, the network model and performance metrics are formally presented. In Sec. III, we analyze the AoI-regret of traditional learning algorithms. In Sec. IV, we develop an order-optimal learning algorithm and analyze its AoI-regret. In Sec. V, we compare the AoI-regret of different learning algorithms using simulations. The paper is concluded in Sec. VI. The technical proofs have been omitted due to the space constraint, and will be made available in a technical report.

\section{System Model}

Consider a single-hop wireless network with $M$ sources, $N$ channels and a single destination, as illustrated in Fig. 1. Each source $m \in\{1, \cdots, M\}$ generates packets containing timesensitive information and these packets are to be transmitted to the destination through one of the wireless channels. Let the time be slotted, with slot index $t \in\{1,2, \cdots, T\}$, where $T$ is the time horizon of this discrete-time system. The slot duration allows for a single packet transmission. We normalize the slot duration to unity.

At the beginning of every slot $t$, each source $m$ generates a packet with probability $\lambda \in(0,1)$. Let $a_{m}(t) \in\{0,1\}$ be the indicator function that is equal to 1 when source $m$ generates 
a packet in slot $t$, and $a_{m}(t)=0$ otherwise. This Bernoulli process with parameter $\lambda$ is i.i.d. over time and independent across different sources, with $\mathrm{P}\left(a_{m}(t)=1\right)=\lambda, \forall m, t$. A packet that is generated in slot $t$ can be transmitted during the same slot $t$. We denote the vector of packet generations in slot $t$ by $\vec{a}(t)=\left[\begin{array}{llll}a_{1}(t) & \cdots & a_{M}(t)\end{array}\right]^{\top}$.

Each source has a transmission queue to store its packets. Sources keep only the most recently generated packet, i.e. the freshest packet, in their queue. When source $m$ generates a new packet at the beginning of slot $t$, older packets (if any) are discarded from its queue. Notice that delivering the most recently generated packet provides the freshest information to the destination. This queueing discipline is known to optimize the AoI in a variety of contexts [21]-[23]. After a packet delivery from source $m$, the queue remains empty until the next packet generation from the same source. However, while the queue is empty, a dummy packet can be transmitted for the purpose of probing the channels.

The networked system is empty during slot $t$ if there are no data packets available for transmission, i.e. if the $M$ queues are empty. Let $E(t) \in\{0,1\}$ be the indicator function that is equal to 1 if the system is empty during slot $t$, and $E(t)=0$ otherwise. Notice that if there is a packet generation at the beginning of slot $t$, then the system is nonempty during slot $t$ and $E(t)=0$. Recall that when the system is empty, sources can still transmit dummy packets.

In a slot, the learning algorithm selects a single pair $(m, n)$, where $m \in\{1,2, \cdots, M\}$ is the index of the source and $n \in\{1,2, \cdots, N\}$ is the index of the wireless channel. Then, during this slot, source $m$ transmits a packet to the destination through channel $n$. If channel $n$ is $\mathrm{ON}$, then the packet is successfully transmitted to the destination, and if channel $n$ is OFF, then the transmission fails. The learning algorithm does not know the channel state while making scheduling decisions, and the outcome of a transmission attempt during slot $t$ is known at the beginning of slot $t+1$. Let $b_{n}(t) \in\{0,1\}$ be the indicator function that represents the state of channel $n$ during slot $t$. The channel is $\mathrm{ON}, b_{n}(t)=1$, with probability $\mu_{n} \in(0,1]$, and the channel is $\mathrm{OFF}, b_{n}(t)=0$, with probability $1-\mu_{n}$. The channel state process is i.i.d. over time and independent across different channels.

The reliability of channel $n$ is represented by the probability of this channel being $\mathrm{ON}, \mu_{n}$. Let $\vec{\mu}=\left[\begin{array}{lll}\mu_{1} & \cdots & \mu_{N}\end{array}\right]^{\top}$ be the vector of channel reliabilities. Let $\mu^{*}$ be the maximum channel reliability and let $n^{*}$ be the index of the corresponding channel, i.e. $\mu^{*}=\max _{n} \mu_{n}=\mu_{n^{*}}$. For simplicity, we assume that the optimal channel $n^{*}$ is unique. Naturally, if the channel reliabilities were known by the learning algorithm in advance, then it would select channel $n^{*}$ in every slot $t$. However, since the channel reliabilities $\vec{\mu}$ are initially unknown, the learning algorithm has to estimate $\mu_{n}$ using observations from previous transmission attempts, while at the same time attempting to minimize the AoI in the network. Next, we formulate the AoI minimization problem.

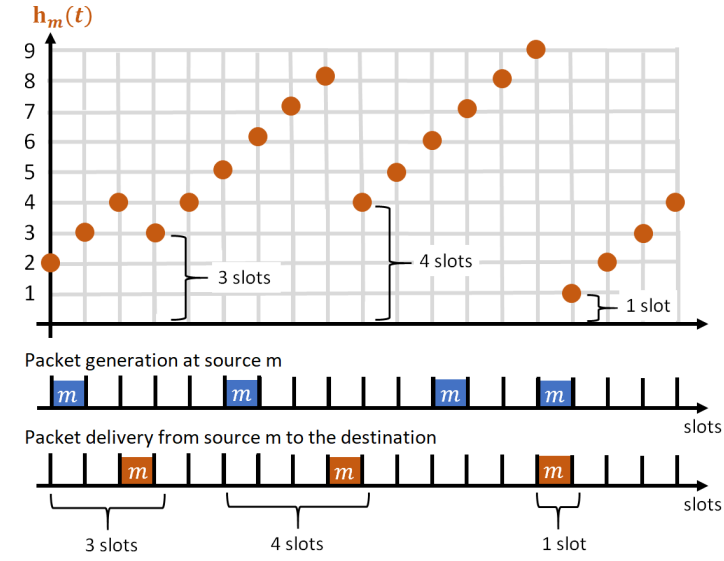

Fig. 2. The blue and orange rectangles at the bottom represent packets generated at source $m$ and successful packet transmissions from source $m$ respectively. The orange curve shows the AoI evolution $h_{m}(t)$ associated with source $m$. Recall that each of the $M$ sources has its own AoI evolution.

\section{A. Age of Information}

The AoI captures how old the information is from the perspective of the destination. Let $h_{m}(t)$ be a positive integer that represents the AoI associated with source $m$ at the beginning of slot $t$. By definition, we have $h_{m}(t):=t-\tau_{m}(t)$, where $\tau_{m}(t)$ is the generation time of the latest packet successfully transmitted from source $m$ to the destination ${ }^{1}$. If the destination does not receive a fresh packet from source $m$ during slot $t$, then in the next slot we have $h_{m}(t+1)=h_{m}(t)+1$, since the information at the destination is one slot older. In contrast, if the destination receives a fresh packet from source $m$ during slot $t$, then in the next slot the value of $\tau_{m}(t+1)$ is updated to the generation time of the received packet and the AoI is reduced by $\tau_{m}(t+1)-\tau_{m}(t)$. This difference is the "freshness gain" associated with the received packet. The evolution of $h_{m}(t)$ over time is illustrated in Fig. 2. We define the vector of AoI in slot $t$ as $\vec{h}(t)=\left[\begin{array}{lll}h_{1}(t) & \cdots & h_{M}(t)\end{array}\right]^{\top}$.

For capturing the information freshness of the entire network, we consider the expected total age $\bar{h}(T)$, which is defined as the expected sum of the AoI over all sources and over time, namely

$$
\bar{h}(T)=\mathrm{E}\left[\sum_{m=1}^{M} \sum_{t=1}^{T} h_{m}(t)\right],
$$

where the expectation is with respect to the randomness in the channel states $b_{n}(t)$, packet generation process $\vec{a}(t)$, and scheduling decisions $(m, n)$. The learning algorithm schedules pairs $(m, n)$ over time so as to minimize the expected total AoI $\bar{h}(T)$. Recall that in this sequential decision problem, the channel reliabilities $\mu_{n}$ are initially unknown by the learning algorithm and should be estimated over time. Next, we discuss the class of learning algorithms considered in this paper.

\section{B. Learning Algorithm}

In this section, we present three important concepts associated with the learning algorithm: the channel policy, the

\footnotetext{
${ }^{1}$ We define $\tau_{m}(t)=0$ prior to the first packet delivery from source $m$.
} 
source policy, and the AoI-regret. Prior to discussing these concepts, we introduce some notation. In each slot $t$, the learning algorithm selects a single source and a single channel. Let $m(t)$ be the index of the source selected during slot $t$ and let $n(t)$ be the index of the channel selected during slot $t$. Then, the pair selected in slot $t$ can be denoted as $(m(t), n(t))$. Notice that the learning algorithm consists of a source policy, which selects $m(t)$, and a channel policy, which selects $n(t)$. Let $b(t)=b_{n(t)}(t)$ be the state of the channel selected during slot $t$, and recall that $\vec{a}(t)$ is the vector of packet generations and $\vec{h}(t)$ is the vector of AoI in slot $t$. Next, we use this notation to define the channel policy and the source policy.

The channel policy may (or may not) take into account the status of the transmission queues at the sources (in particular $E(t))$ in making scheduling decisions $n(t)$. Hence, we define two types of channel policies: queue-independent channel policies and queue-dependent channel policies. Let $\Pi_{B}$ be the class of admissible queue-independent channel policies $\pi_{b}$. In slot $t$, an arbitrary policy $\pi_{b} \in \Pi_{B}$ selects $n(t)$ using information about the outcome of previous transmission attempts. In particular, the queue-independent channel history in slot $t$ is given by $H_{B}(t)=\{n(1), b(1), \ldots, n(t-1), b(t-1)\}$. Let $\bar{\Pi}_{B}$ be the class of admissible queue-dependent channel policies $\bar{\pi}_{b}$. In slot $t$, an arbitrary policy $\bar{\pi}_{b} \in \bar{\Pi}_{B}$ selects $n(t)$ using information about the outcome of previous transmission attempts and about the current status of the transmission queues. In particular, the queue-dependent channel history in slot $t$ is given by $\bar{H}_{B}(t)=H_{B}(t) \cup\{E(t)\}$. In Sec. IV, we show that this small amount of information, namely $E(t)$, can have a significant impact on the performance of the channel policy. It is easy to see that both the optimal queue-independent channel policy $\pi_{b}^{*}$ and the optimal queuedependent channel policy $\bar{\pi}_{b}^{*}$ select, in every slot $t$, the channel $n^{*}$ with highest reliability $\mu^{*}$. However, since the reliabilities $\vec{\mu}$ are not known a priori, the channel policies have to estimate $\vec{\mu}$ over time. In Sec. III, we consider queue-independent channel policies and in Sec. IV, we consider queue-dependent channel policies.

The source policies considered in this paper are workconserving, i.e. policies that never transmit dummy packets when there are undelivered data packets in the system. Let $\Pi_{A}$ be the class of admissible work-conserving source policies $\pi_{a}$. In slot $t$, an arbitrary source policy $\pi_{a} \in \Pi_{A}$ selects $m(t)$ using information about the current AoI and the generation times of the packets waiting to be transmitted at the sources' queues. In particular, the source history in slot $t$ is given by $H_{A}(t)=\{\vec{a}(1), \vec{h}(1), \ldots, \vec{a}(t), \vec{h}(t)\}$. The optimal source policy $\pi_{a}^{*} \in \Pi_{A}$ is the transmission scheduling policy that minimizes the expected total AoI in (1). A few works in the literature [14], [15], [24] have addressed the problem of finding the transmission scheduling policy that minimizes AoI in wireless networks with stochastic packet generation and unreliable channels with known statistics. Despite those efforts, a full characterization of the optimal source policy is still an open problem.

In this paper, we consider learning algorithms $\pi=\left(\pi_{a}, \pi_{b}\right)$ that are composed of a source policy and a channel policy. Our goal is to study the exploration-exploitation dilemma faced by the channel policy. To that end, we analyze the AoI-regret of learning algorithms employing the optimal source policy and different channel policies. To analyze the AoI-regret of learning algorithms without the full characterization of the optimal source policy $\pi_{a}^{*}$, we derive lower and upper bounds on the regret. These bounds are discussed in Proposition 2, Proposition 3, and Theorem 7, where we assumed that the optimal source policy $\pi_{a}^{*}$ is the same irrespective of the queueindependent channel policy $\pi_{b}$ under consideration, namely

$$
\pi_{a}^{*}=\underset{\pi_{a} \in \Pi_{A}}{\arg \min } \mathrm{E}\left[\sum_{m=1}^{M} \sum_{t=1}^{T} h_{m}^{\left(\pi_{a}, \pi_{b}\right)}(t)\right], \forall \pi_{b} \in \Pi_{B},
$$

where $h_{m}^{\left(\pi_{a}, \pi_{b}\right)}(t)$ denotes the AoI associated with source $m$ in slot $t$ when the learning algorithm $\pi=\left(\pi_{a}, \pi_{b}\right)$ is employed. An analogous assumption is utilized for the case of queuedependent channel policies $\bar{\pi}_{b} \in \bar{\Pi}_{B}$.

Clearly, the assumption in (2) does not hold in general. For example, if we consider a channel policy that purposefully selects poor wireless channels when a particular source $m^{\prime}$ is selected, then this channel policy would create an incentive for the optimal source policy to avoid selecting $\mathrm{m}^{\prime}$, thus contradicting the assumption in (2). However, for the channel policies considered in this paper, which employ information about the outcomes of previous transmissions in order to attempt to select the most reliable channel, irrespective of the selected source, there seems to be no incentive for the optimal source policy to change its strategy based on the channel policy, which is in line with the assumption in (2).

The AoI-regret of a learning algorithm $\pi$ with queueindependent channel policy $\pi_{b}$ is defined as the difference between the expected total AoI $\bar{h}^{\pi}(T)$ when $\pi=\left(\pi_{a}^{*}, \pi_{b}\right)$ is employed and the expected total AoI $\bar{h}^{*}(T)$ when the optimal algorithm $\pi^{*}=\left(\pi_{a}^{*}, \pi_{b}^{*}\right)$ is employed, namely

$$
R^{\pi}(T)=\mathrm{E}\left[\sum_{m=1}^{M} \sum_{t=1}^{T} h_{m}^{\pi}(t)-\sum_{m=1}^{M} \sum_{t=1}^{T} h_{m}^{*}(t)\right] .
$$

The definition of AoI-regret for a learning algorithm $\bar{\pi}$ with queue-dependent channel policy $\bar{\pi}_{b}$ is analogous to (3). Next, we analyze the AoI-regret of learning algorithms with queueindependent channel policies.

\section{REGRET ANALYSIS}

The problem of learning channel reliabilities over time is closely related to the stochastic MAB problem. A natural class of channel policies to consider are traditional MAB algorithms such as $\epsilon$-Greedy, UCB, and TS. In this section, we derive bounds on the AoI-regret of learning algorithms that employ queue-independent channel policies. Notice that the class of queue-independent channel policies $\Pi_{B}$ includes traditional MAB algorithms. We describe a learning algorithm employing TS as its channel policy in Algorithm 1.

Scheduling decisions of a learning algorithm $\pi$ might differ from those of $\pi^{*}$ both in the source and in the channel, which 


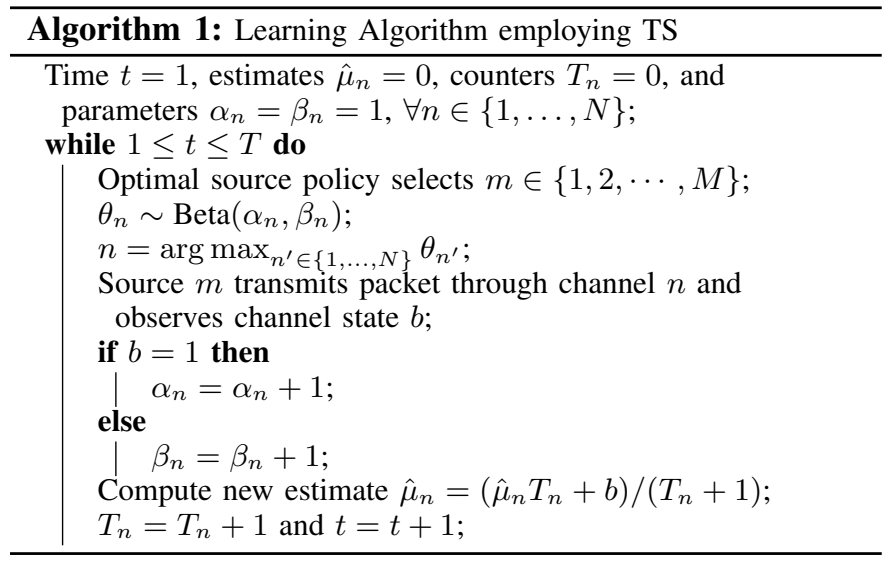

makes the analysis of the AoI-regret $\sum_{t=1}^{T} \sum_{m=1}^{M} \mathrm{E}\left[h_{m}^{\pi}(t)-\right.$ $\left.h_{m}^{*}(t)\right]$ challenging. To alleviate this challenge, we use stochastic coupling to create equivalent coupled channel state processes that are simpler to analyze. Similar coupling arguments were employed in [10], [18].

Remark 1 (Coupled Channel States). Let $\{U(t)\}_{t=1}^{T}$ be a sequence of i.i.d. random variables uniformly distributed in the interval $[0,1]$. In each slot $t$, the channel states $b_{n}(t)$ are determined as follows: $b_{n}(t)=1 \Longleftrightarrow 0 \leq U(t) \leq \mu_{n}, \forall n$.

By construction, the coupled channel states are no longer independent. In particular, if a channel is ON during slot $t$, then all channels with higher reliability $\mu_{n}$ are also ON during that slot. Notice that, in each slot $t$, each coupled channel $n$ has the same probability distribution as the associated original channel $n$, namely $\mathrm{P}\left(b_{n}(t)=1\right)=\mu_{n}, \forall n, t$. Hence, given the scheduling decision $(m(t), n(t))$ of $\pi$ during any slot $t$, the probability of a successful transmission attempt from source $m(t)$ through channel $n(t)$ is the same for both the coupled and original channel states. It follows that the probability distribution of $h_{m}^{\pi}(t)$ also remains the same for all slots $t$ and for all sources $m$ and, thus, the AoI-regret $R^{\pi}(T)$ in (3) also remains the same for both the coupled and original channel state processes. For simplicity of analysis, henceforth in this paper, we assume that the channel state processes are coupled as described in Remark 1.

In Propositions 2 and 3 and Corollary 4, we derive bounds on the AoI-regret of a learning algorithm $\pi$ with respect to its expected number of suboptimal channel choices, namely

$$
\mathrm{E}\left[K^{\pi}(T)\right]=\mathrm{E}\left[\sum_{t=1}^{T} \mathbb{1}_{\left\{n^{\pi}(t) \neq n^{*}\right\}}\right]
$$

where $\mathbb{1}_{\left\{n^{\pi}(t) \neq n^{*}\right\}}=1$ if $n^{\pi}(t) \neq n^{*}$, and $\mathbb{1}_{\left\{n^{\pi}(t) \neq n^{*}\right\}}=$ 0 otherwise. We consider two classes of admissible learning algorithms

$$
\begin{gathered}
\Pi=\left\{\pi=\left(\pi_{a}, \pi_{b}\right): \pi_{a} \in \Pi_{A}, \pi_{b} \in \Pi_{B}\right\} \\
\Pi^{*}=\left\{\pi=\left(\pi_{a}, \pi_{b}\right): \pi_{a}=\pi_{a}^{*}, \pi_{b} \in \Pi_{B}\right\} .
\end{gathered}
$$

Both classes employ queue-independent channel policies. The difference is that learning algorithms in $\Pi$ employ any admissible source policy $\pi_{a} \in \Pi_{A}$, while learning algorithms in $\Pi^{*}$ employ the optimal source policy $\pi_{a}^{*}$. Naturally, we have that $\Pi^{*} \subset \Pi$.

Proposition 2 (Lower Bound). For any given network configuration $(\lambda, \vec{\mu})$, the AoI-regret of any learning algorithm $\pi \in \Pi$ scales at least on the order of its expected number of suboptimal channel choices, namely ${ }^{2} R^{\pi}(T)=\Omega\left(\mathrm{E}\left[K^{\pi}(T)\right]\right)$.

Proof outline. In addition to the suboptimal channel choices, source choices $m^{\pi}(t)$ of algorithm $\pi \in \Pi$ can also differ from the source choices $m^{*}(t)$ of $\pi^{*}$. To overcome this challenge, we construct an auxiliary algorithm $\hat{\pi}^{*}$ with optimal channel policy and a source policy that selects the same source ${ }^{3} m^{\pi}(t)$ as $\pi$ in every slot $t$. Then, we focus on the auxiliary AoI-regret $\sum_{t=1}^{T} \sum_{m=1}^{M} \mathrm{E}\left[h_{m}^{\pi}(t)-h_{m}^{\hat{\pi}^{*}}(t)\right]$ associated with the auxiliary algorithm $\hat{\pi}^{*}$, which we show to be not greater than the original AoI-regret $\sum_{t=1}^{T} \sum_{m=1}^{M} \mathrm{E}\left[h_{m}^{\pi}(t)-h_{m}^{*}(t)\right]$. We then observe that each suboptimal channel choice of $\pi$ results in a penalty to the auxiliary AoI-regret, and we show that this penalty is lower bounded by a constant. Using this constant, we obtain the desired lower bound on the original AoI-regret. The details are omitted due to the space constraint.

Proposition 3 (Upper Bound). For any given network configuration $(\lambda, \vec{\mu})$, the AoI-regret of any learning algorithm $\pi \in \Pi^{*}$ scales at most on the order of its expected number of suboptimal channel choices, namely ${ }^{4}(T)=O\left(\mathrm{E}\left[K^{\pi}(T)\right]\right)$.

Proof outline. Despite the fact that both learning algorithms $\pi \in \Pi^{*}$ and $\pi^{*}$ employ the same optimal source policy $\pi_{a}^{*}$, they might select different sources $m^{\pi}(t) \neq m^{*}(t)$ over time, due to their different channel policies. To address this challenge, we use an approach similar to the proof of Proposition 2. We construct an auxiliary algorithm $\hat{\pi} \in \Pi^{*}$ with a source policy that selects the same source $m^{*}(t)$ as $\pi^{*}$ in every slot $t$, and with a channel policy that selects the same channel $n^{\pi}(t)$ as $\pi$ in every slot $t$. Then, we show that the auxiliary AoI-regret $\sum_{t=1}^{T} \sum_{m=1}^{M} \mathrm{E}\left[h_{m}^{\hat{\pi}}(t)-h_{m}^{*}(t)\right]$ associated with the auxiliary algorithm $\hat{\pi}$ is not lower than the original AoI-regret $\sum_{t=1}^{T} \sum_{m=1}^{M} \mathrm{E}\left[h_{m}^{\pi}(t)-h_{m}^{*}(t)\right]$. To derive an upper bound on the auxiliary AoI-regret, we analyze the penalty that results from each suboptimal channel choice of $\hat{\pi}$. During a slot $t$ where $\hat{\pi}$ makes a suboptimal channel choice, if channel $n^{\hat{\pi}}(t)$ is OFF and channel $n^{*}$ is $\mathrm{ON}$, then a discrepancy is added to the difference between the AoI of $\hat{\pi}$ and the AoI of $\pi^{*}$, i.e. $h_{m}^{\hat{\pi}}(t+1)-h_{m}^{*}(t+1)>h_{m}^{\hat{\pi}}(t)-h_{m}^{*}(t)$. This discrepancy lasts until the next successful transmission of a packet from source $m$ by the auxiliary algorithm $\hat{\pi}$, after which the values of $h_{m}^{\hat{\pi}}(\cdot)$ and $h_{m}^{*}(\cdot)$ become equal ${ }^{5}$. We refer to the duration of the discrepancy as its length. The penalty that results from a suboptimal channel choice is the product of the discrepancy and its length. We characterize the auxiliary AoI-

\footnotetext{
${ }^{2} f(t)=\Omega(g(t)) \Longleftrightarrow \exists C>0 \exists t_{0} \forall t>t_{0}: f(t) \geq C \cdot g(n)$

${ }^{3}$ Notice that if the selected source $m^{\pi}(t)$ has no packet in its transmission queue, then the auxiliary algorithm attempts to transmit a dummy packet.

${ }^{4} f(t)=O(g(t)) \Longleftrightarrow \exists C>0 \exists t_{0} \forall t>t_{0}: f(t) \leq C \cdot g(n)$

${ }^{5}$ Recall from Remark 1 that channel states are coupled. Hence, if channel
} $n^{\hat{\pi}}(t)$ is ON, then channel $n^{*}$ is also ON. 
regret by expressing it as the sum of the penalties arising from suboptimal channel choices. Then, using discrete phase-type distributions, we upper bound the discrepancies and the lengths by constants (in the expected sense) to obtain the desired upper bound on the original AoI-regret. The details are omitted due to the space constraint.

Corollary 4. For any given network configuration $(\lambda, \vec{\mu})$, the AoI-regret of any learning algorithm $\pi \in \Pi^{*}$ scales with its expected number of suboptimal channel choices, namely ${ }^{6}$ $R^{\pi}(T)=\Theta\left(\mathrm{E}\left[K^{\pi}(T)\right]\right)$.

Corollary 4 follows directly from Proposition 2 and Proposition 3. Notice that the bounds in Proposition 3 and Corollary 4 are not valid for the broader class of learning algorithms $\Pi$ which includes suboptimal source policies. This is because suboptimal source choices may add to the AoI-regret, possibly making it grow faster than $\mathrm{E}\left[K^{\pi}(T)\right]$.

Prior to analyzing the AoI-regret of learning algorithms that employ $\epsilon$-Greedy, UCB, and TS as their channel policy, we define $\alpha$-consistent learning algorithms [9], [10] and discuss a few of their properties. Let $\mathrm{E}\left[T_{n}^{\pi}(T)\right]$ be the expected number of times that channel $n$ is selected by $\pi \in \Pi$ in the first $T$ slots, namely

$$
\mathrm{E}\left[T_{n}^{\pi}(T)\right]=\mathrm{E}\left[\sum_{t=1}^{T} \mathbb{1}_{\left\{n^{\pi}(t)=n\right\}}\right] .
$$

Definition 5 ( $\alpha$-consistency). For a given $\alpha \in(0,1)$, a learning algorithm $\pi \in \Pi$ is classified as $\alpha$-consistent if, for any network configuration $(\lambda, \vec{\mu})$, we have $E\left[T_{n}^{\pi}(T)\right]=O\left(T^{\alpha}\right)$ for all suboptimal channels $n \neq n^{*}$.

Intuitively, a learning algorithm $\pi \in \Pi$ is $\alpha$-consistent if its channel policy has good performance in every network configuration. Consider a learning algorithm with a trivial channel policy that selects $n(t)=1$ in every slot $t$. In network configurations with $n^{*}=1$, this channel policy never selects suboptimal channels, i.e. $\mathrm{E}\left[T_{n}^{\pi}(T)\right]=O\left(T^{\alpha}\right), \forall n \neq n^{*}$. However, in network settings with $n^{*} \neq 1$, this channel policy always selects suboptimal channels, i.e. $\mathrm{E}\left[T_{1}^{\pi}(T)\right]=T$, which violates the definition of $\alpha$-consistency. In the remainder of this section, we focus on channel policies that have good performance in every network configuration. In particular, we analyze the AoI-regret of $\alpha$-consistent learning algorithms with queue-independent channel policies.

Remark 6 (AoI-regret of $\alpha$-consistent algorithms). In [10, Corollary 20], the authors show that any learning algorithm $\pi \in \Pi$ that is $\alpha$-consistent has an expected number of suboptimal channel choices that scales as $E\left[K^{\pi}(T)\right]=\Omega(\log T)$, for any network configuration $(\lambda, \vec{\mu})$. Hence, it follows from the lower bound in Proposition 2 that the associated AoI-regret scales as $R^{\pi}(T)=\Omega(\log T)$, for any network configuration $(\lambda, \vec{\mu})$.

Notice that the lower bound in Remark 6 applies to $\alpha$ consistent learning algorithms with queue-independent chan-

\footnotetext{
${ }^{6} f(t)=\Theta(g(t)) \Longleftrightarrow f(t)=O(g(t)) \wedge f(t)=\Omega(g(t)) \Longleftrightarrow$ $\exists C_{1}, C_{2}>0 \exists t_{0} \forall t>t_{0}: C_{1} \cdot g(n) \leq f(t) \leq C_{2} \cdot g(n)$
}

nel policies that do not know the statistics of the channels in advance.

Learning algorithms that employ $\epsilon$-Greedy, UCB, and TS as their channel policy are known to have suboptimal channel choices scaling as $\mathrm{E}\left[K^{\pi}(T)\right]=O(\log T)$ for any network configuration $(\lambda, \vec{\mu})$ [3], [25], which implies that they are $\alpha$-consistent. Hence, it follows from the lower bound in Remark 6 and from the upper bound in Proposition 3 that the AoI-regret of these traditional MAB algorithms scale as $R^{\pi}(T)=\Theta(\log T)$.

In [18], the authors derived lower and upper bounds on the AoI-regret of learning algorithms employing queueindependent channel policies, including UCB and TS, in networks with a single source generating and transmitting fresh packets in every slot $t$. Propositions 2 and 3 generalize the results in [18] to networks with multiple sources generating packets according to stochastic processes. The analysis of the AoI-regret is more challenging in this network setting for the following reasons: i) the optimal source policy $\pi_{a}^{*}$ is unknown and there is no closed-form expression for the expected total AoI (1) of the optimal algorithm $\pi^{*}=\left(\pi_{a}^{*}, \pi_{b}^{*}\right)$; and ii) the learning algorithm under consideration $\pi=\left(\pi_{a}, \pi_{b}\right)$ can make suboptimal choices both in terms of sources $m(t)$ and channels $n(t)$, and these two types of suboptimal choices affect the AoI-regret $R^{\pi}(T)$ differently. Next, we develop a learning algorithm that leverages information about the status of the transmission queues at the sources in making scheduling decisions $n(t)$, and show that this new learning algorithm has $O(1)$ AoI-regret.

\section{ORder-Optimal Learning AlgORithm}

In this section, we develop a learning algorithm $\bar{\eta} \in \bar{\Pi}$ with a queue-dependent channel policy that selects $n(t)$ using information about the outcome of previous transmission attempts, namely $H_{B}(t)=\{n(1), b(1), \cdots, n(t-1), b(t-1)\}$, and about the current status of the transmission queues, namely $E(t)$. Then, we derive an upper bound on its AoI-regret. In particular, we show that the AoI-regret of $\bar{\eta}$ is such that $R^{\bar{\eta}}(T)=O(1)$. Notice that the only difference between the learning algorithms $\pi \in \Pi$ considered in Sec. III and the orderoptimal learning algorithm $\bar{\eta}$ is the knowledge of $E(t)$. This seemingly modest addition led to the reduction of the AoIregret from $R^{\pi}(T)=\Omega(\log T)$ to $R^{\bar{\eta}}(T)=O(1)$. To the best of our knowledge, this is the first learning algorithm with bounded AoI-regret.

The key insight is that when packets are generated randomly, the learning algorithm $\bar{\eta}$ can utilize times when the network has no data packets to transmit, i.e. when $E(t)=1$, to transmit dummy packets and learn the statistics of the channels without incurring an opportunity cost The orderoptimal learning algorithm $\bar{\eta}=\left(\eta_{a}, \bar{\eta}_{b}\right)$ has optimal source policy $\eta_{a}=\pi_{a}^{*}$ and a channel policy $\bar{\eta}_{b} \in \bar{\Pi}_{B}$ that operates as follows: when the system is empty, $E(t)=1$, the policy chooses a channel uniformly at random and uses the outcome of the transmission attempt to update its estimates of the channel reliabilities and, when the system is nonempty, 


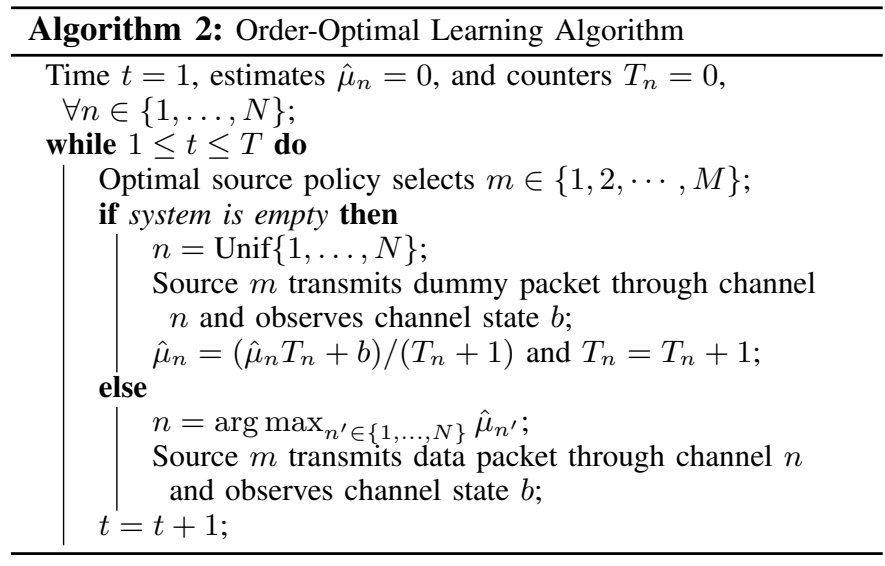

$E(t)=0$, the policy chooses the channel with the current highest estimated reliability. Notice that the channel policy only updates its estimates of the channel reliabilities when the system is empty. A similar channel policy was used in [11], [12] to develop a learning algorithm with bounded queue-length regret. The order-optimal learning algorithm $\bar{\eta}$ is described in Algorithm 2. The upper bound on the AoI-regret is established in the theorem that follows.

Theorem 7. For any given network configuration $(\lambda, \vec{\mu})$, the AoI-regret of the order-optimal learning algorithm $\bar{\eta}$ is bounded, namely $R^{\bar{\eta}}(T)=O(1)$.

Proof outline. Recall from Algorithm 2 that estimates of the channel reliabilities $\hat{\mu}_{n}$ are only updated when the system is empty $E(t)=1$. Hence, during nonempty periods, the channel reliabilities do not change and, thus, the selected channel also does not change. First, we show that $\bar{\eta}$ only contributes to the AoI-regret when it selects a suboptimal channel during a nonempty period. Then, we derive an upper bound on the AoI-regret by analyzing: i) the evolution of the probability of $\bar{\eta}$ selecting a suboptimal channel during a nonempty period; and ii) the corresponding contribution to the AoI-regret. To bound the probability, we employ Hoeffding's inequality. To bound the contribution to the AoI-regret, we use discrete phasetype distributions. From these two upper bounds, we obtain $R^{\bar{\eta}}(T)=O(1)$. The details are omitted due to the space constraint.

In the particular case of a network with sources generating fresh packets at every slot $t$, i.e. $\lambda=1$, the algorithm $\bar{\eta}$ cannot utilize slots in which the system is empty to learn the channel reliabilities without incurring a cost in terms of AoI-regret, which results in a $R^{\bar{\eta}}(T)$ that grows over time. The upper bound in Theorem 7 is only valid for the network models described in Sec. II, in which $\lambda \in(0,1)$. Next, we evaluate the AoI-regret of the different learning algorithms discussed in this paper using MATLAB simulations and we propose a heuristic algorithm that leverages both the fast learning rates of TS and the bounded regret of the order-optimal algorithm.

\section{Simulations}

In this section, we evaluate the performance of learning algorithms in terms of the AoI-regret in (3). We compare learning algorithms employing the Age-Based Max-Weight source policy [24, Sec. 5] and different channel policies, namely: i) $\epsilon$-Greedy; ii) UCB; iii) TS; iv) Optimal; and v) Hybrid. The Age-Based Max-Weight source policy selects, in each slot $t$, the source $m$ associated with the packet that gives the largest AoI reduction, $\tau_{m}(t+1)-\tau_{m}(t)$, if the transmission in slot $t$ is successful. Intuitively, this policy is selecting the source with highest potential reward in terms of AoI. In [24], we evaluated the performance of the AgeBased Max-Weight source policy both analytically and using simulations, and showed that it achieves near optimal AoI. The first three channel policies, namely $\epsilon$-Greedy, UCB, and TS, were discussed in Sec. III. The Optimal policy is the order-optimal channel policy $\bar{\eta}_{b}$ developed in Sec. IV. The Hybrid policy employs TS for a fixed period in the beginning of the simulation and then employs the Optimal policy in the remaining slots.

We simulate a network with a time horizon of $T=5 \times 10^{5}$ slots, $M=3$ sources, each generating packets according to a Bernoulli process with rate $\lambda$, and $N=5$ channels with reliabilities $\vec{\mu}=\left[\begin{array}{lllll}0.4 & 0.45 & 0.5 & 0.55 & 0.6\end{array}\right]^{\top}$. Figures 3 (a) and 3(b) show simulation results of the evolution of the AoI-regret over time for $\lambda=0.1$ and $\lambda=0.7$, respectively. Figure 3(c) shows the evolution of the reliability estimates associated with the channels with $\mu_{4}=0.55$ and $\mu_{5}=0.6$ over time for $\lambda=0.7$. Figure 3(d) shows the AoI-regret at the last slot of the simulation, i.e., $R^{\pi}\left(T=5 \times 10^{5}\right)$, for increasing values of $\lambda \in\{0.01,0.1,0.2, \cdots, 0.9\}$. Each data point in Figs. 3(a)3 (d) is an average over the results of 200 simulations.

The results in Figs. 3(a) and 3(b) suggest that, as expected, the AoI-regrets associated with Optimal and Hybrid are bounded, while the AoI-regrets associated with $\epsilon$-Greedy, $\mathrm{UCB}$ and TS grow over time. By comparing the AoI-regrets of the different channel policies in Fig. 3(d), it becomes clear that the AoI-regret of Optimal varies significantly with $\lambda$. In particular, for $T=5 \times 10^{5}$, when $\lambda$ increases from 0.1 to 0.7 , the AoI-regret of TS increases by a factor of 1.6 (from 1,583 to 2,505), while the AoI-regret of Optimal increases by a factor of $1,123.8$ (from 1,176 to $1,321,580$ ). A main reason for this performance degradation is that when $\lambda$ increases, empty systems with $E(t)=1$ occur less often and, as a result, the Optimal channel policy takes longer to learn the reliability of the channels. However, it is important to emphasize that, for any $\lambda \in(0,1)$ and for a long enough time-horizon $T$ : (i) the Optimal channel policy will eventually converge to the true reliabilities, as can be seen in Fig. 3(c), at which point its AoI-regret will stop increasing; and (ii) the AoI-regret of $\epsilon$-Greedy, UCB and TS will never stop increasing and, thus, at some moment, their performance will become worse than the Optimal policy.

To improve the performance of the Optimal policy for networks with large $\lambda$, we propose a heuristic policy called Hybrid channel policy, which employs TS in the first $5 \times 10^{4}$ slots to quickly learn the reliability of the channels, and then shifts to the Optimal policy which has bounded AoI-regret in the long term. Figure 3(c) illustrates the difference in the 


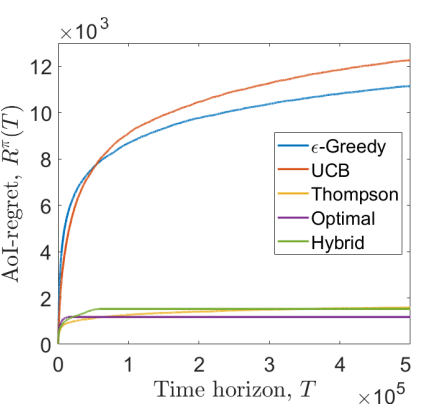

(a) AoI-regret for $\lambda=0.1$

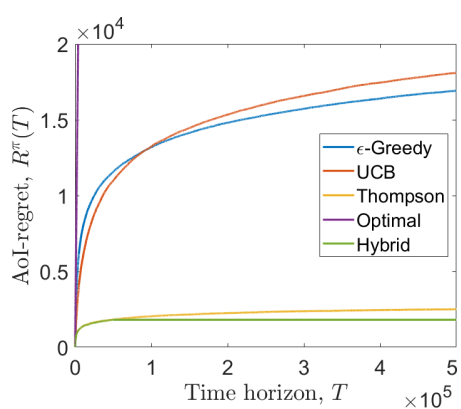

(b) AoI-regret for $\lambda=0.7$

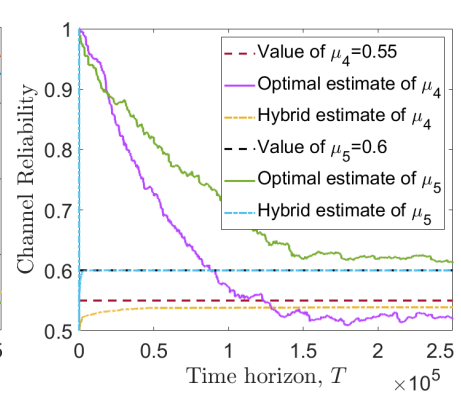

(c) Reliability for $\lambda=0.7$

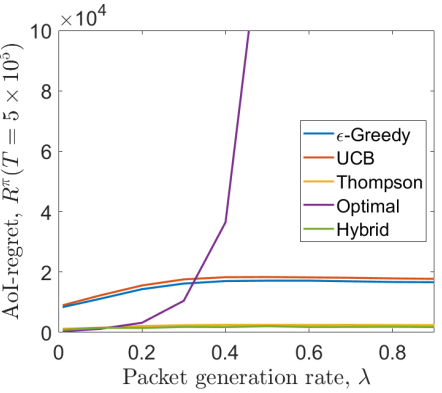

(d) AoI-regret at the last slot

Fig. 3. Simulation of networks with different $\lambda$. In (a) and (b), we show the evolution of the AoI-regret over time. In (c), we show the evolution of the reliability estimates over time. In (d), we show the AoI-regret at the end of the simulation for increasing values of $\lambda$.

learning rates between Optimal and Hybrid. Notice in Fig. 3(c) that there are extended periods of time in which Optimal assigns a larger estimated reliability to a suboptimal channel, which leads to the larger AoI-regret shown in Figs. 3(b) and 3(d). This learning period is significantly shorter with Hybrid.

\section{CONCLUSION}

This paper considers a single-hop wireless network with $M$ sources transmitting time-sensitive information to the destination over $N$ unreliable channels. Packets from each source are generated according to a Bernoulli process with known rate $\lambda$ and the state of channel $n$ (ON/OFF) varies according to a Bernoulli process with unknown rate $\mu_{n}$. The reliabilities $\vec{\mu}$ of the wireless channels is to be learned through observation. At every slot $t$, the learning algorithm selects a single pair $(m(t), n(t))$ and the selected source $m(t)$ attempts to transmit its packet via the selected channel $n(t)$. The goal of the learning algorithm is to minimize the expected total AoI $\bar{h}(T)$. To analyze the performance of the learning algorithm, we derive bounds on the AoI-regret $R^{\pi}(T)$ associated with different learning algorithms. Our main contributions include: i) analyzing the performance of learning algorithms that employ channel policies based on traditional MAB algorithms $(\epsilon-$ Greedy, UCB, and TS) and showing that their AoI-regret scales as $\Theta(\log T)$; and ii) developing a novel learning algorithm and establishing that it has $O(1)$ AoI-regret. To the best of our knowledge, this is the first learning algorithm with bounded AoI-regret. Interesting extensions of this work include consideration of sources with unknown packet generation rates and channels with time-varying statistics.

\section{ACKNOWLEDGMENT}

This work was supported by NSF Grant CNS-1713725 and by Army Research Office (ARO) grant number W911NF-17$1-0508$.

\section{REFERENCES}

[1] A. Kosta, N. Pappas, and V. Angelakis, "Age of information: A new concept, metric, and tool," Foundations and Trends in Networking, vol. 12, no. 3, pp. 162-259, 2017.

[2] Y. Sun, I. Kadota, R. Talak, and E. Modiano, Age of Information: A New Metric for Information Freshness. Morgan \& Claypool, 2019.

[3] P. Auer, N. Cesa-Bianchi, and P. Fischer, "Finite-time analysis of the multiarmed bandit problem," Machine Learning, vol. 47, no. 2-3, p. 235-256, May 2002.
[4] A. Garivier and O. Cappé, "The KL-UCB algorithm for bounded stochastic bandits and beyond," in Proc. of COLT, 2011.

[5] S. Agrawal and N. Goyal, "Analysis of thompson sampling for the multiarmed bandit problem," in Proc. of COLT, 2012.

[6] T. Lai and H. Robbins, "Asymptotically efficient adaptive allocation rules," Advances in Applied Mathematics, vol. 6, no. 1, pp. 4-22, 1985.

[7] W. R. Thompson, "On the likelihood that one unknown probability exceeds another in view of the evidence of two samples," Biometrika, vol. 25, pp. 285-294, 1933.

[8] A. Slivkins, "Introduction to multi-armed bandits," Foundations and Trends in Machine Learning, vol. 12, pp. 1-286, 2019.

[9] S. Bubeck and N. Cesa-Bianchi, "Regret analysis of stochastic and nonstochastic multi-armed bandit problems," Foundations and Trends in Machine Learning, vol. 5, pp. 1-122, 2012.

[10] S. Krishnasamy, R. Sen, R. Johari, and S. Shakkottai, "Learning unknown service rates in queues: A multiarmed bandit approach," Operations Research, vol. 69, no. 1, pp. 315-330, 2020.

[11] T. Stahlbuhk, B. Shrader, and E. Modiano, "Learning algorithms for minimizing queue length regret," in Proc. of IEEE ISIT, 2018.

[12] T. B. Stahlbuhk, "Control of wireless networks under uncertain state information," Ph.D. dissertation, MIT, 2018.

[13] I. Kadota, A. Sinha, E. Uysal-Biyikoglu, R. Singh, and E. Modiano, "Scheduling policies for minimizing age of information in broadcast wireless networks," IEEE/ACM Transactions on Networking, vol. 26, no. 6, pp. 2637-2650, 2018.

[14] Y.-P. Hsu, "Age of information: Whittle index for scheduling stochastic arrivals," in Proc. of IEEE ISIT, 2018.

[15] Y.-P. Hsu, E. Modiano, and L. Duan, "Scheduling algorithms for minimizing age of information in wireless broadcast networks with random arrivals," IEEE Transactions on Mobile Computing, vol. 19, no. 12, pp. 2903-2915, 2020.

[16] J. Sun, Z. Jiang, B. Krishnamachari, S. Zhou, and Z. Niu, "Closed-form whittle's index-enabled random access for timely status update," IEEE Transactions on Communications, vol. 68, no. 3, pp. 1538-1551, 2020.

[17] V. Tripathi and E. Modiano, "A whittle index approach to minimizing functions of age of information," in Proc. of IEEE Allerton, 2019.

[18] K. Bhandari, S. Fatale, U. Narula, S. Moharir, and M. K. Hanawal, "Age-of-information bandits," in Proc. of IEEE WiOpt, 2020.

[19] I. Juneja, S. Fatale, and S. Moharir, "Correlated age-of-information bandits," arXiv preprint, arXiv:2011.05032, 2020.

[20] A. Prasad, V. Jain, and S. Moharir, "Decentralized age-of-information bandits," arXiv preprint, arXiv:2009.12961, 2020.

[21] M. Costa, M. Codreanu, and A. Ephremides, "On the age of information in status update systems with packet management," IEEE Transactions on Information Theory, vol. 62, no. 4, pp. 1897-1910, 2016.

[22] S. Kaul, R. D. Yates, and M. Gruteser, "Status updates through queues," in Proc. of IEEE CISS, 2012.

[23] A. M. Bedewy, Y. Sun, and N. B. Shroff, "The age of information in multihop networks," IEEE/ACM Transactions on Networking, vol. 27, no. 3, pp. 1248-1257, 2019.

[24] I. Kadota and E. Modiano, "Minimizing the age of information in wireless networks with stochastic arrivals," IEEE Transactions on Mobile Computing, 2019.

[25] S. Agrawal and N. Goyal, "Further optimal regret bounds for thompson sampling," in Proc. of AISTATS, 2013. 